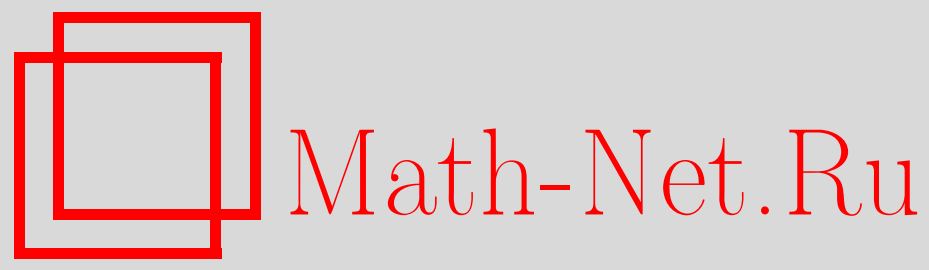

В. Д. Седых, Теорема о четырех опорных вершинах ломаной, Функи. анализ и его прил., 1996, том 30, выпуск 3, 88-90

DOI: https://doi.org/10.4213/faa542

Использование Общероссийского математического портала Math$\mathrm{Net.Ru}$ подразумевает, что вы прочитали и согласны с пользовательским соглашением

http://www . mathnet.ru/rus/agreement

Параметры загрузки:

IP : 54.237 .206 .68

26 апреля 2023 г., 17:55:45

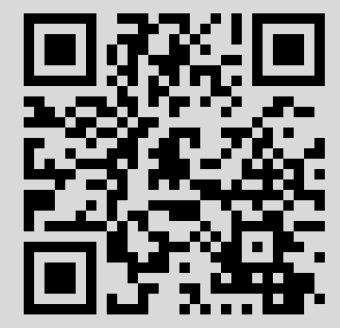




\title{
Теорема о четырех опорных вершинах ломаной
}

\author{
(C) 1996. В. Д. СЕдыХ ${ }^{1}$
}

Для выпуклого многоугольника справедливо утверждение, аналогичное теореме о четырех вершинах плоской кривой (см. [1]). В настоящей заметке дается дискретный аналог теоремы [3] о четырех вершинах выпуклой пространственной кривой. В качестве следствия получено обобщение теоремы из [1] на случай невыпуклых многоугольников.

1. Опорные вершины ломаных в пространстве. Ломаной называется непрерывная локально простая кривая, состоящая из конечного числа отрезков. Эти отрезки называются звеньями ломаной, а их концы - вериинами.

Рассмотрим замкнутую ломаную в пространстве. Плоскость, проходящая через одну или несколько ее вершин, называется опорной, если ломаная лежит от нее с одной стороны.

ОПРЕДЕЛЕНИЕ. Вершина ломаной называется опорной, если через нее и две соседние вершины проходит опорная плоскость.

Выпуклой оболочкой ломаной называется пересечение всех замкнутых полупространств, ее содержащих. Выпуклая оболочка ломаной, не лежащей в плоскости, — это выпуклый многогранник, вершины которого являются вершинами ломаной.

ОПРЕДЕЛЕНИЕ. Ломаная называется выпуклой, если она лежит на границе своей выпуклой оболочки.

Рассмотрим неплоскую замкнутую выпуклую ориентированную несамопересекающуюся ломаную в ориентированном пространстве. Всякие три ее последовательные вершины, не лежащие на одной прямой, являются вершинами (невырожденного) треугольника, ориентированного обходом вершин в соответствии с ориентацией ломаной.

ОПРЕДЕЛЕНИЕ. Опорная вершина ломаной называется правой (левой), если она и две ее соседние вершины определяют невырожденный треугольник, ориентированный против часовой стрелки (по часовой стрелке) относительно нормали к нему, внешней по отношению к выпуклой оболочке этой ломаной.

ТЕОРема 1. Всякая неплоская замкнутая несамопересекаюшаяся выпуклая ориентированная ломаная в пространстве имеет не менее двух правых и двух левых опорных вершин.

Доказательство аналогично доказательству теоремы [3].

СЛЕдСТВИЕ 1. Замкнутая несамопересекающаяся выпуклая $n$-звенная ломаная в пространстве с $n>3$ имеет не менее четырех опорных вериин.

Рассмотрим пять последовательных вершин $A, B, C, D, E$ ломаной в пространстве.

ОПРЕДЕЛЕНИЕ. Вершина $C$ называется вершиной уплощения ломаной, если вершины $A$ и $E$ лежат с одной стороны от плоскости, проходящей через вершины

${ }^{1}$ Работа поддержана Российским фондом фундаментальных исследований (проект 9401-01203) и Международной ассоциацией INTAS (грант 4373). 
$B, C$ и $D$ (если таких плоскостей много, то от одной из них).

Если никакие четыре последовательные вершины замкнутой ломаной с числом звеньев, большим трех, не лежат в одной плоскости, то число ее вершин уплощения четно.

СЛЕдСТВИЕ 2. Замкнутая несамопересекаюшаяся выпуклая $n$-звенная ломаная в пространстве с $n>3$ имеет не менее четьрех вершин уплощения.

2. Опорные вершины плоских многоугольников. Рассмотрим многоугольник (замкнутую ломаную) на плоскости. Окружность (прямая), проходящая через одну или несколько его вершин, называется опорной, если все вершины многоугольника лежат от окружности (прямой) с одной стороны.

ОПРЕДЕЛЕНИЕ. Вершина многоугольника называется опорной, если окружность (или прямая), проходящая через нее и две соседние вершины, является опорной.

ОПРЕДЕЛЕНИЕ. Многоугольник называется регулярнылм, если через любые две его соседние вершины можно провести опорную окружность.

Рассмотрим несамопересекающийся ориентированный многоугольник на ориентированной плоскости. Его ориентация индуцирует ориентацию каждой опорной окружности или прямой, проходяшей через три последовательные вершины. Предположим, что многоугольник не вписывается в окружность.

ОПРЕДЕЛЕНИЕ. ОПорная вершина многоугольника называется правой (левой), если через нее и две соседние вершины проходит опорная окружность или прямая, от которой вершины многоугольника лежат слева (справа).

ТЕОРема 2. Всякий несамопересекающийся регулярный ориентированньй многоугольник на плоскости, не вписываюшийся в окружность, имеет не менее двух правых и двух левых опорных вершин.

Доказательство состоит в применении теоремы 1 к ломаной в пространстве, полученной из стереографического образа многоугольника (см. [4]) заменой образа каждой стороны многоугольника отрезком, соединяющим образы концов этой стороны.

СЛЕДСТВИЕ 3. Всякий несамопересекающийся регулярньй $n$-угольник на плоскости с $n>3$ имеет не менее четьирех опорных вериин.

Для каждой вершины $V$ несамопересекающегося ориентированного многоугольника можно определить кривизну $\kappa(V)$ по следующему правилу. Если вершина $V$ и две ее соседние вершины $V^{\prime}, V^{\prime \prime}$ лежат на одной прямой, то $\kappa(V)=0$. В противном случае $\kappa(V)= \pm 1 / R$, где $R$ - радиус окружности, описанной вокруг треугольника $\triangle V^{\prime} V V^{\prime \prime}$, и выбирается знак $+(-)$, если ориентация многоугольника индуцирует ориентацию треугольника $\triangle V^{\prime} V V^{\prime \prime}$ против часовой стрелки (по часовой стрелке).

ОПРЕДЕЛЕНИЕ. Вершина многоугольника называется экстремальной, если его кривизна в этой вершине одновременно либо не меньше, либо не больше кривизны в обеих соседних вершинах.

Если никакие четыре последовательные вершины многоугольника с числом сторон, большим трех, не лежат на одной окружности или прямой, то число его экстремальных вершин четно.

ОПРЕДЕЛЕНИЕ. Несамопересекающийся многоугольник на плоскости называется неособым, если центр окружности, проведенной через любые три после- 
довательные его вершины $A, B, C$, не лежащие на одной прямой, расположен внутри угла $\angle A B C$.

СЛЕДСТВИЕ 4. Неособый регулярный многоугольник на плоскости с числом сторон, больиим трех, имеет не менее четьиех экстремальньх вершин.

Это утверждение является прямым обобщением теоремы [1] на случай невыпуклых многоугольников.

ОПРЕДЕЛЕНИЕ. Опорная вершина $V$ плоского многоугольника называется внутренней (внешней), если некоторые его вершины лежат внутри (вне) опорной окружности, проходящей через вершину $V$ и две ее соседние.

СЛЕДСТВИЕ 5 [6]. Выпукльй многоугольник на плоскости, не вписывающийся в окружность, имеет не менее двух внутренних и двух внешних вершин.

СЛЕДСТВИЕ 6. Любой несамопересекающийся регулярный многоугольник на плоскости либо вписьвается в окружность, либо имеет не менее двух внешних вершин.

Имеются и другие варианты теоремы о четырех вершинах для многоугольника (обзор см., например, в [7]). Автор благодарен О. Р. Мусину, заметившему, что условие регулярности плоского несамопересекающегося многоугольника эквивалентно тому, что этот многоугольник является подграфом триангуляиии Делоне множества своих вершин (см. [2, 5]).

\title{
ЛИтеРАТУРА
}

1. Мусин О. Р. Квант (1996) (в печати). 2. Препарата Ф., Шеймос М. Вычислительная геометрия: Введение. Мир, М., 1989. 3. Седых В. Д. Функц. анализ и его прил., 26, вып. 1, 35-41 (1992). 4. Седыл В. Д. Функц. анализ и его прил., 29, вып. 3, 41-50 (1995). 5. Guibas L., Stolfi J. ACM Trans. Graphics., 4, 74-123 (1985). 6. Schatteman A. Geom. Dedicata, 34, 229-242 (1990). 7. Wegner B. Math. Pannon., 6, No. 1, 121-132 (1995).

Московский государственный технологический университет «СТАНКИН»

Поступило в редакцию 25 декабря 1995 г.

\section{Векторные и ковекторные инварианты супералгебр Ли}

\author{
(C) 1996. A. H. СерГеЕB
}

Пусть $V$ - конечномерное суперпространство над $\mathbb{C}$ и $\mathfrak{g}$ - подсупералгебра Ли в $\mathfrak{g l}(V)$. В работе приводится описание образующих алгебр $\mathfrak{g}$-инвариантных элементов, содержащихся в $\mathfrak{A}_{k, l}^{p, q}=S\left(V^{k} \oplus \pi(V)^{l} \oplus V^{* p} \oplus \pi\left(V^{*}\right)^{q}\right)$. Полученные результаты являются обобщением работы [3], где аналогичное описание дано с точностью до операторов поляризации.

1. Инварианты супералгебры Ли $\mathrm{sl}(\boldsymbol{V})$. Для заданного суперпространства $E$ через $B(E)$ обозначим множество $\{1, \ldots, \alpha, \overline{1}, \ldots, \bar{\beta}\}$, где $\alpha=\operatorname{dim} E_{\overline{0}}$, 